\title{
Transient Myocardial Dysfunction of Newborn
}

National Cancer Institute

\section{Source}

National Cancer Institute. Transient Myocardial Dysfunction of Newborn. NCI Thesaurus.

Code C113156.

A condition in a newborn characterized by a temporary decrease in right ventricular and/or left ventricular output. 